\title{
Analytics for Info-plosion Including Information Diffusion Studies for the 3.11 Disaster
}

\author{
Masaru Kitsuregawa and Masashi Toyoda \\ Institute of Industrial Science, The University of Tokyo, \\ 4-6-1 Komaba Meguro-ku, 1538505 Tokyo, Japan \\ \{kitsure, toyoda\} atkl.iis.u-tokyo.ac.jp
}

\begin{abstract}
Information explosion(Info-plosion) is one of the most substantial phenomena in 21st century. Not only the mentions in blogs and twitter, but also the data from various kinds of sensors is becoming to explode considerably. In Japan, we conducted national project, Info-plosion by MEXT(Ministry of Education, Sports, Culture, Science and Technology), Grand Infomation Voyage by METI(Ministry of Economy, Trade and Industry). Recently we started FIRST project. In this talk, we would like to show how info-plosion analytics, especially sensor analytics, created disruptive services. In addition, we will show the diffusion pattern analysis of twitter and blogs for the 3.11 disaster. On 9.11, we did not have real time media such as microblogging, while such media quite effectively worked on 3.11.

Explosive amount of information is becoming available, especially since around the beginning of 21 st century. This causes so called information overload. Although there are such negative problems, we could think that this is a first experience for human being to see such a vast amount of information. Taking advantage of such a totally new opportunity, we believe various disruptive services could be introduced which we have never imagined a decade ago. We launched info-plosion project in 2005. CPS started to get funding since 2009, while its idea was discussed in 2006. IOT, smarter planet, M2M, and Big Data etc. are targetting not necessarily exactly the same objective, but the goals are quite similar. We would like to introduce some of our experiments. We collected Japanese blogs and tweets in March 11th earthquake and tsunami disaster. Although mobile phone service stopped, the Internet was not damaged. Lots of interesting societal movement happened immediately after the earthquake such as refuge place notification, power saving etc. We examined its diffusion pattern. In addition, we also could clarify the difference of role among the media. People are using blog and microblog differently. We will report the role of IT media and its importance.
\end{abstract}

